\title{
Type I Brugada-Like Pattern with Arrhythmia in Severe Anemia
}

\author{
Wilson Saputra Wijaya (1)', Ika Krisnawati \\ 'Faculty of Medicine, Trisakti University, Jakarta, Indonesia; ${ }^{2}$ Department of Cardiology, Tangerang City General Hospital, Tangerang City, Indonesia \\ Correspondence: Wilson Saputra Wijaya, Faculty of Medicine, Trisakti University, Jakarta, Indonesia, Tel +62 8I I I I3 938, Email werewilsonsw@gmail.com
}

Introduction: Brugada syndrome $(\mathrm{BrS})$ has been described as an inherited cardiac disorder due to gene mutations, which contributes to sudden cardiac death, especially in Southeast Asians. Brugada phenocopy, which has the same pattern caused by etiologies such as metabolic disorder, is potentially reversible and avoids unnecessary implantable medical devices.

Case Presentation: We reported a rare case of Brugada phenocopy in a 60-years-old Indonesian male with severe anemia and a history of chronic urinary tract bleeding and hemorrhoid. Brugada phenocopy, widely known as BrS look-alike, has been linked to sodium, potassium, and calcium channel dysfunction. This condition is uncommon and has been related to metabolic conditions such as anemia. Iron deficiency anemia induces ischemia in the myocardium and degrades the calcium channels. During the transfusion, hypocalcemia precipitates temporary arrhythmia with right ventricular outflow tract origin, which reverts after the hypocalcemia has been treated.

Conclusion: Due to the possibility of altered ion channels and how rarely hypocalcemia induces arrhythmia, this makes understanding the pathogenesis of Brugada phenocopy essential. Though caution is necessary, early recognition can improve prognosis as Brugada phenocopy is potentially reversible.

Keywords: Brugada phenocopy, severe anemia, calcium channel, right ventricular outflow tract

\section{Introduction}

Brugada syndrome (BrS) has been described as an inherited cardiac disorder due to gene mutations without ischemia, carditis, congenital heart diseases and electrolyte imbalance. These mutations can be precipitated by fever, vagal tone, drugs, and alcohol, leading to palpitations, syncope, malignant tachycardia, and sudden cardiac death. The annual incidence of syncope due to arrhythmia was between 0.5 to $4 \%$ in BrS. Southeast Asians are at an increased risk of BrS, which was estimated at $\geq 3.5$ per 1000 person, with Thailand at 14 times higher than worldwide prevalence. ${ }^{1-3}$

Preventive measurement for precipitation factors is required and, sometimes, treatment with implantable cardioverterdefibrillator is necessary to prevent malignant tachycardia. ${ }^{1}$ However, heterogeneous conditions such as metabolic disorder (ie, hyperkalemia, hypokalemia, toxicities), ischemia, pericarditis, and many other causes are able to induce Brugada patterns. These conditions have been described as Brugada phenocopy (BrP). ${ }^{1,4}$ Identifying BrP is essential, as they can be treated as reversible causes; hence invasive treatment can be avoided and quality of life for the patients improved.

\section{Case Presentation}

A 60-year-old Indonesian male presented with malaise and fatigue for a week, without prior chest pain, fever, dyspnea, syncope, or diarrhea, and was admitted to the emergency unit. Before his hospitalization, he had recurrent hematuria with blood clots and a history of bloody feces for a month. He was hospitalized for abdominal pain with hematuria in another hospital around two months ago. He was previously diagnosed with hematuria from an intravesical urinary lesion, suspected of malignant origin that obstructed vesica urinary right junction, complicated with mild hydronephrosis, and multiple renal cysts in the left. He was scheduled for an intravesical biopsy, but he never came to the appointment. He was afebrile with blood pressure $127 / 72 \mathrm{mmHg}$, a heart rate of 102 times per minute, and normal oxygen saturation. We 
found pallid conjunctival, pallid skin, with normal heart and lung sounds during the physical examination. We performed a digital rectal examination and found internal hemorrhoid grade II.

Twelve-lead electrocardiography (ECG) showed sinus tachycardia with a "coved" morphology ST-segment in V1 and V2 similar to Brugada type-I pattern with early repolarization in V3 (Figure 1). The patient did not have a family history of sudden cardiac arrest, syncopal attack, or other heart diseases. Sodium channel blocking agents such as ajmaline, flecainide, and procainamide were not available; hence we did not perform a provocative challenge. AP chest radiography indicated a normal cardiac size without lung infiltrates.

At admission, the hematology test showed hemoglobin $(\mathrm{Hb})$ level $3.1 \mathrm{~g} / \mathrm{dL}$ with hematocrit level $12 \%$ and a slight leukocytosis. In addition, blood urea $84 \mathrm{mg} / \mathrm{dL}$ and creatinine $2.0 \mathrm{mg} / \mathrm{dL}$, while partial thromboplastin, activated partial thromboplastin time, and electrolytes level remained normal. Therefore, we concluded this patient's case as a highly suspected Brugada phenocopy (BrP) induced by metabolic disorder due to severe anemia with acute kidney injury caused by intravesical urinary lesion and internal hemorrhoid. During his inpatient stay, he received packed red cells transfusion for $500 \mathrm{cc}$ each day until hemoglobin level reached $10.0 \mathrm{~g} / \mathrm{dL}$. He was scheduled for a urology computed tomography (CT) scan.

Another 12-lead ECG was performed on the second day and revealed bigeminy premature ventricular contraction (PVC) consistent with a right ventricular outflow tract (RVOT) origin (Figure 2A). Another electrolyte blood test showed mild hypocalcemia with a normal magnesium level, so we added an extra calcium gluconate intravenous 2 gr followed by another ECG in the morning (Figure 2B). Another calcium gluconate intravenous 1 gr was added with bisoprolol $2.5 \mathrm{mg}$ once daily the next day. As the renal function did not improve, an abdominal CT scan without contrast was performed instead. CT scan revealed suspected intravesical mass with bilateral hydronephrosis grade II and bilateral hydroureter. His anemia improved, with hemoglobin level 9.7g/dL. ECG was performed with the evolution of V1 and V2; the "coved" morphology resolved, a new J-point appeared similar with the "saddleback" morphology of Brugada and occasional PVCs with the same prior morphology (Figure 2B).

After a hemorrhoidectomy, followed by transurethral resection of the bladder tumor, another PRC transfusion was given. The biopsy concluded as a papillary urothelial neoplasm of low malignant potential (PUNLMP). The patient was permitted to go home with hemoglobin level $10.6 \mathrm{~g} / \mathrm{dL}$. ECG reverted to sinus rhythm and prominent J-point consistent with early repolarization in V2-V4 (Figure 3) from the fourth day until he returned home.

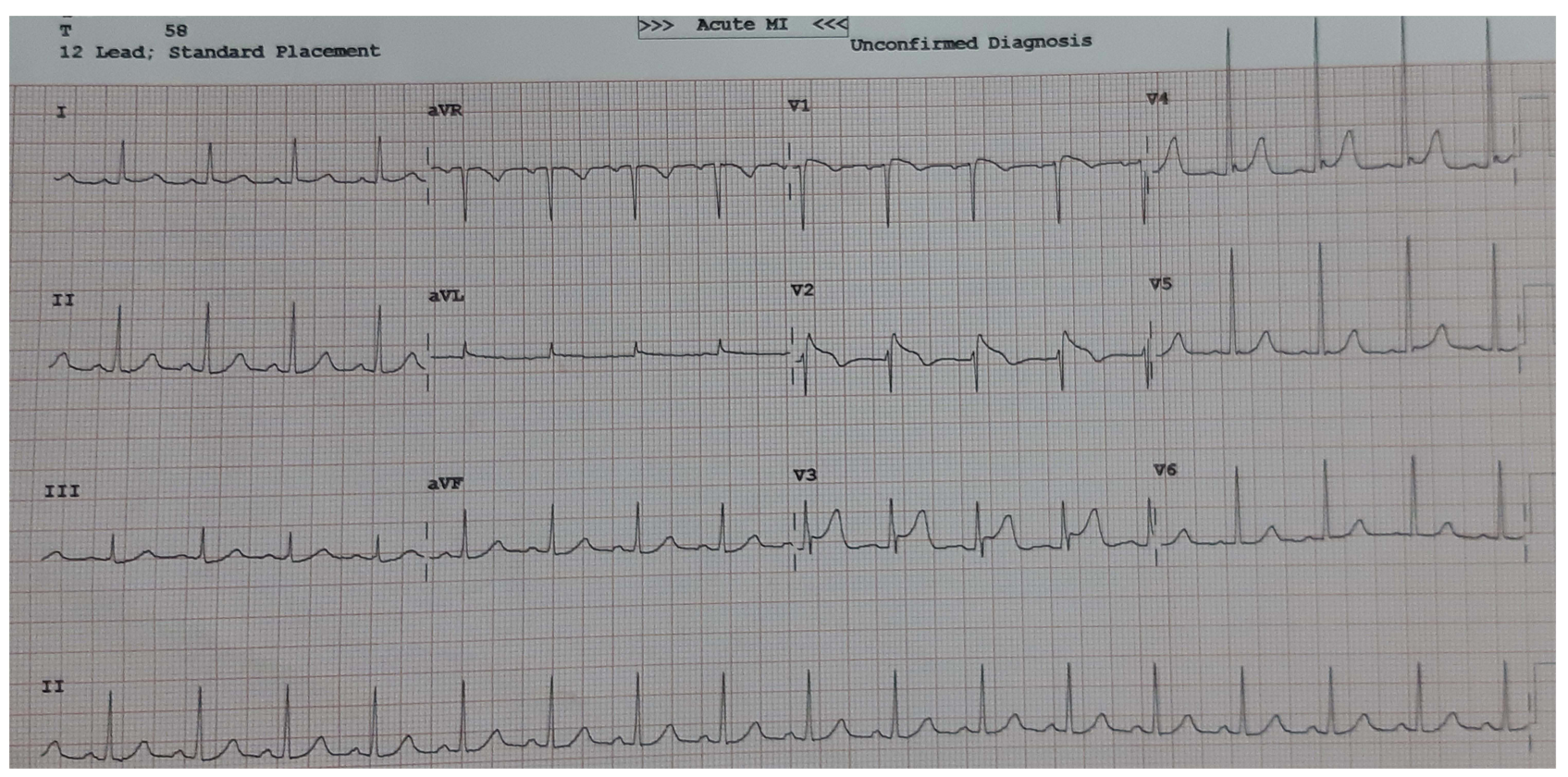

Figure I ECG during admission showed "coved" morphology similar to Brugada type I pattern with early repolarization in V3. 

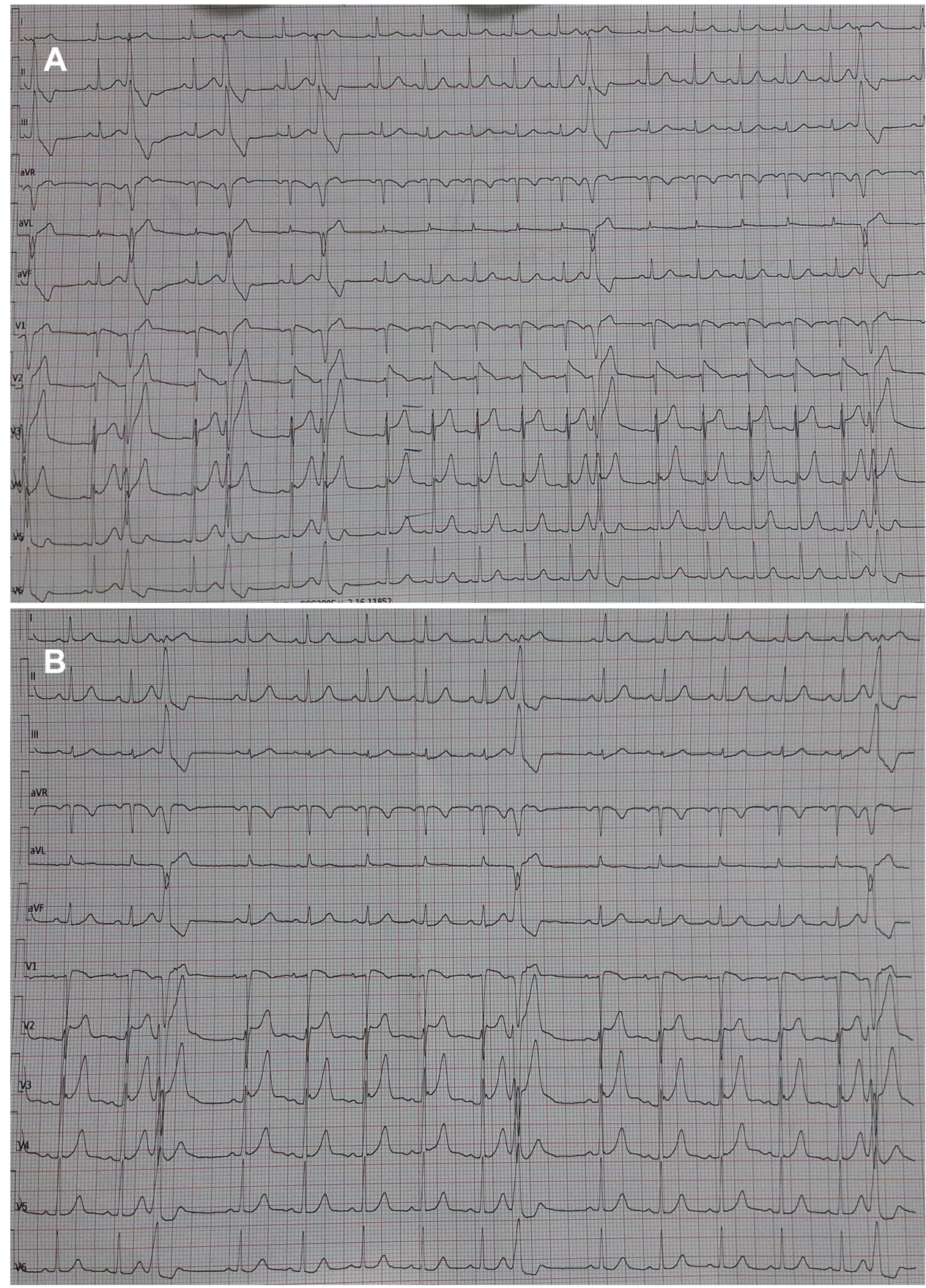

Figure 2 ECG before calcium supplementation (A) showing bigeminy PVC suspected RVOT origin. The next day, after calcium supplementation and blood transfusions (B), the ECG showed "saddleback" morphology of Brugada with occasional PVCs.

\section{Discussion}

Brugada syndrome (BrS) has been known as a dominant genetic condition that leads to tachyarrhythmias and sudden cardiac death. This heredity, primarily due to loss of function in SCN5A or other genes, a predominantly male inheritance, induces cardiac sodium channelopathy, and possibly substrate due to structural changes. ${ }^{5,6}$ Sodium channelopathy in $\mathrm{BrS}$ alters the function of sodium $\left(\mathrm{Na}^{+}\right)$, potassium $\left(\mathrm{K}^{+}\right)$, calcium $\left(\mathrm{Ca}^{2+}\right)$, and hyperpolarization-activated cyclic nucleotide-gated (HCN) channels. Reduced sodium current availability in Phase 0 is associated with reduced ionic exchange across the cell membrane. Reduced function in L-type calcium channels, leads to reduced calcium influx in 


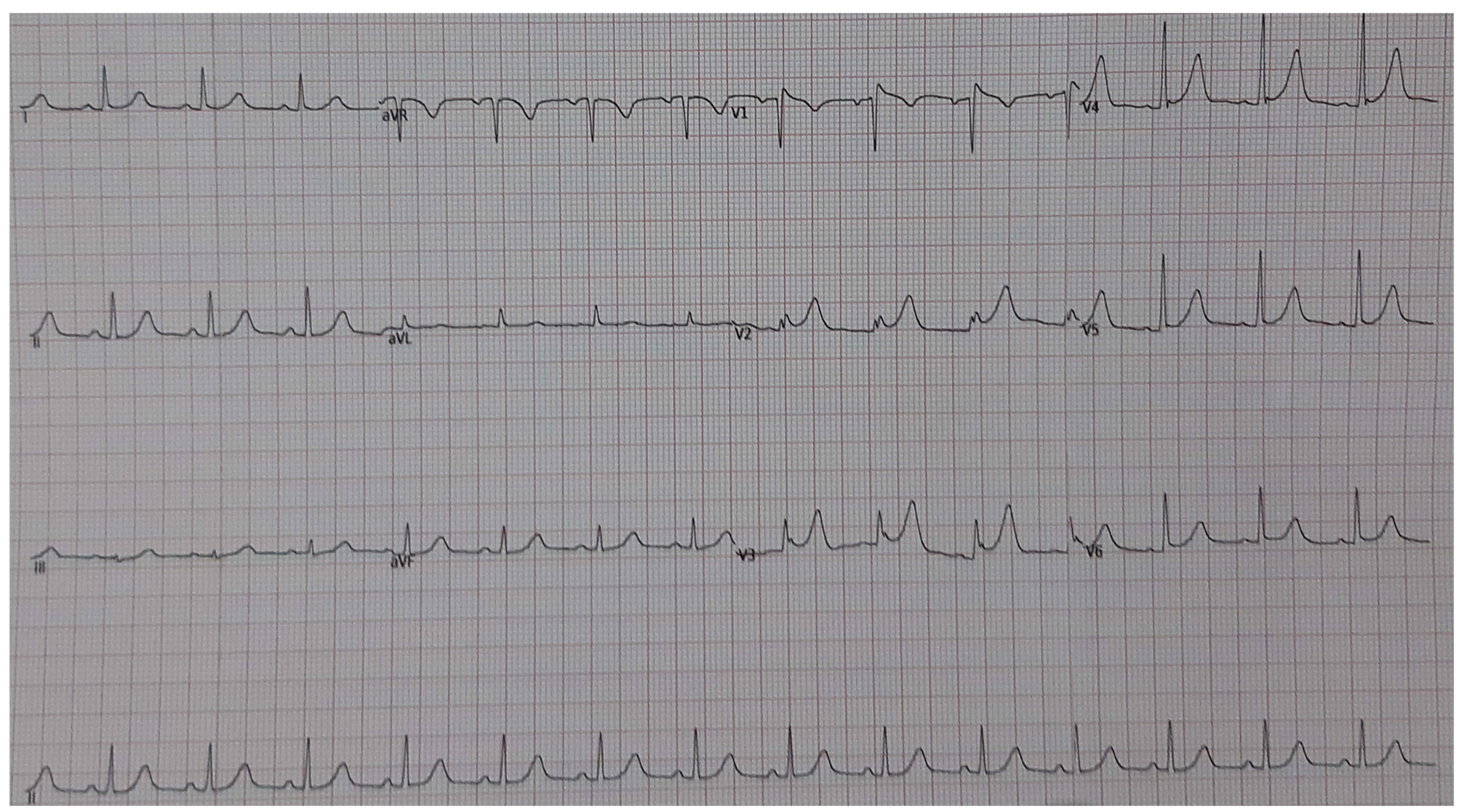

Figure 3 ECG on the fourth day.

Phase 2. The pathogenic gene and cellular abnormalities induce RVOT inflammation, structural changes, and conduction delay, leading to substrate for Brugada pattern. Aging also contributes to RVOT remodeling. ${ }^{1,6}$

Brugada phenocopy $(\mathrm{BrP})$ is known as clinical entities which are characterized by Brugada pattern (type 1 and type 2), with different etiology. Etiologies can vary from ischemia, myocardial and pericardial disease, mechanical compression, ECG modulation, and metabolic conditions such as anemia. ${ }^{4,7}$ Patterns showed V1-V2 type 1 "coved" with a high ST-segment elevation $\geq 2 \mathrm{~mm}$ followed by a negative symmetric T-wave (Figure 1). In comparison, type 2 "saddleback" morphology has high r' ( $\geq 2 \mathrm{~mm}$ from baseline), followed by convex ST-segment with elevation $\geq 0.05 \mathrm{mV}$ from baseline and positive or flat T-wave in lead V2 (Figure 2B). ${ }^{4-6}$

Anemia has been known to cause ECG changes due to hemoglobin's physiological role in delivering oxygen to vital organs, especially the heart. In one study with 31,898 patients, 21 patients with severe anemia $(\mathrm{Hb}<6 \mathrm{~g} / \mathrm{dL})$ showed small QRS amplitude, rightward T-axis deviation, and longer corrected QT interval, and one of the patients reverted to normal after transfusion. ${ }^{8}$ Another study, by Khatri et al, ${ }^{9}$ in 300 patients with moderate $(<10 \mathrm{~g} / \mathrm{dL})$ and severe $(<7 \mathrm{~g} / \mathrm{dL})$ anemia, showed changes in ECG, especially ST-segment. Five of them are ST-segment elevation. Mismatching in oxygen demand and supply in the myocardium leads to an increase in cardiac output, heart rate, cardiac size (either hypertrophy or dilatation), also ECG changes. All these changes reportedly return to normal in a few weeks after the anemia resolves. ${ }^{9,10}$

The relation between $\mathrm{BrP}$ and anemia has been reported in Kattenbeck et al, ${ }^{7}$ but its mechanism remains unclear due to only a limited number of severe anemia cases with $\mathrm{BrP}$. Another known cause of $\mathrm{BrP}$ is synthetic cannabinoid usage, which induces corrected QT prolongation and potential inhibitory effect in sodium channels. ${ }^{5}$ One of the possible mechanisms is disturbance in ion transportation caused by severe anemia. Iron deficiency anemia has increased red cell membrane $\mathrm{Na}^{+}-\mathrm{K}^{+}-\mathrm{Cl}^{-}$ATPase activity that leads to hyponatremia. ${ }^{11} \mathrm{PVC}$ in this patient is similar to RVOT origin as described by Behr et al, ${ }^{6}$ elucidating patient BrP originates possibly from the substrate in RVOT, which is provoked by metabolic disturbances.

Calcium plays an important role in inducing arrhythmias and contributes to BrS. Degradation in the L-type $\mathrm{Ca}^{2+}$ channel, mainly ryanodine receptors (RyR2) and Sarco/endoplasmic reticulum $\mathrm{Ca}^{2+}$-ATPase (SERCA) activity, decreases the release and uptake of calcium from the sarcoplasmic reticulum. ${ }^{12,13}$ Iron-deficiency anemia decreases cardiac 
contraction, potentially downregulating RyR2 channel and SERCA pump activity. ${ }^{12}$ Interestingly, arrhythmia appeared possibly due to hypocalcemia during the second transfusion since calcium binds to citrate preservatives in blood products. Arrhythmia in severe hypocalcemia remains rare; in Duval et al, ${ }^{14}$ among 133 cases, three patients showed a cardiac complication but with an underlying severe co-morbidity.

Follow-up serum calcium is not necessary since there are no recurrent arrhythmias. It remains unclear if the anemia can trigger the same ECG pattern as in BrS. With ST-segment elevation remains rare in severe anemia. ${ }^{8,9}$ Another interesting point emerges that requires further investigation concerning RVOT remodeling in patients with BrP. Further tests, including electrophysiology and genetic testing, are necessary for the possibility of existing predisposing factors, channelopathy, or structural abnormality.

\section{Conclusion}

We presented a case of type I Brugada phenocopy induced by severe anemia. Sometimes, BrP can be misunderstood as $\mathrm{BrS}$ and lead to delayed treatment. Though caution is necessary, clinical recognition of anemia, as well as its impact on ion channels, is important. The possibility of RVOT pathology contributing to BrP exists, as seen in the ECG. This information can benefit the outcome as BrP is potentially reversible and possibly contributes to our understanding of the $\mathrm{BrS}$ electrophysiological mechanism. However, further investigation is still required to determine if genetic and structural abnormalities also contribute to this condition.

\section{Ethics Approval}

The case was approved by the Ethical Committee of Tangerang City General Hospital. The patient consented to participate in the study and the surgery procedure. Informed written consent was obtained and signed. Signed informed consent for publication was also obtained from the patient to publish this case report and any accompanying images.

\section{Disclosure}

The authors report no conflicts of interest in this work.

\section{References}

1. Li KHC, Lee S, Yin C, et al. Brugada syndrome: a comprehensive review of pathophysiological mechanisms and risk stratification strategies. IJC Heart Vasc. 2020;26:100468. doi:10.1016/j.ijcha.2020.100468

2. Postema PG. About Brugada syndrome and its prevalence. Europace. 2012;14(7):925-928. doi:10.1093/europace/eus042

3. Vutthikraivit W, Rattanawong P, Putthapiban P, et al. Worldwide prevalence of Brugada syndrome: a systematic review and meta-analysis. Acta Cardiol Sin. 2018;34(3):267-277. doi:10.6515/ACS.201805_34(3).20180302B

4. Gottschalk BH. Brugada phenocopy: definition, diagnosis, and differentiation from true Brugada syndrome. In: Baranchuk A, editor. Brugada Phenocopy: The Art of Recognizing the Brugada ECG Pattern. London: Elsevier; 2018:11-19.

5. Amro M, Okoro KU, Mansoor K, Amro A, Okhumale P. Brugada phenocopy type 1 secondary to synthetic cannabinoids. Case Rep Cardiol. 2020;2020:1-5. doi:10.1155/2020/9425860

6. Behr ER, Ben-Haim Y, Ackerman MJ, Krahn AD, Wilde AAM. Brugada syndrome and reduced right ventricular outflow tract conduction reserve: a final common pathway? Eur Heart J. 2021;42(11):1073-1081. doi:10.1093/eurheartj/ehaa1051

7. Kattenbeck K, Dankl D, Müller-Huesmann H, Odenthal HJ. Brugada-like electrocardiographic pattern induced by an episode of anemia. $Z$ Kardiol. 2005;94(8):537-541. doi:10.1007/s00392-005-0270-x

8. Kwon JM, Cho Y, Jeon KH, et al. A deep learning algorithm to detect anaemia with ECGs: a retrospective, multicentre study. Lancet Digit Health. 2020;2(7):e358-e367. doi:10.1016/S2589-7500(20)30108-4

9. Khatri M, Deokar V, Patel J, Jaju G. Study of electrocardiographic changes in mild, moderate and severe anaemia in a tertiary care hospital. Int J Contemp Med Res. 2018;5(12):9-13.

10. Shashikala GV, Shashidhar PK, Herur A, et al. Correlation between haemoglobin level and electrocardiographic (ECG) findings in anaemia: a cross-sectional study. J Clin Diagn Res. 2014;8(4):6-8.

11. Rajagopal L, Ganesan V, Abdullah S, Arunachalam S, Kathamuthu K, Ramraj B. Perturbations of serum electrolyte levels in iron deficiency anemia - a comparative analysis. Natl J Physiol Pharm Pharmacol. 2018;8(3):370-375.

12. Monasky MM, Pappone C, Piccoli M, Ghiroldi A, Micaglio E, Anastasia L. Calcium in Brugada syndrome: questions for future research. Front Physiol. 2018;9:1088. doi:10.3389/fphys.2018.01088

13. Chung YJ, Luo A, Park KC, et al. Iron-deficiency anemia reduces cardiac contraction by downregulating RyR2 channels and suppressing SERCA pump activity. JCI Insight. 2019;4(7):e125618. doi:10.1172/jci.insight.125618

14. Duval M, Bach-Ngohou K, Masson D, Guimard C, Le Conte P, Trewick D. Is severe hypocalcemia immediately life threatening? Endocr Connect. 2018;7:1067-1074. doi:10.1530/EC-18-0267 


\section{Publish your work in this journal}

The International Medical Case Reports Journal is an international, peer-reviewed open-access journal publishing original case reports from all medical specialties. Previously unpublished medical posters are also accepted relating to any area of clinical or preclinical science. Submissions should not normally exceed 2,000 words or 4 published pages including figures, diagrams and references. The manuscript management system is completely online and includes a very quick and fair peer-review system, which is all easy to use. Visit http://www.dovepress.com/testimonials. php to read real quotes from published authors.

Submit your manuscript here: https://www.dovepress.com/international-medical-case-reports-journal-journal 